\title{
OPTIMAL ADAPTIVE ESTIMATION OF LINEAR FUNCTIONALS UNDER SPARSITY
}

\author{
BY OLIVIER COLlieR ${ }^{*}, 1$, LAËTITIA COMMINGES $^{\dagger}$, \\ AleXANDRE B. TSYBAKOV ${ }^{\ddagger}, 2$ AND NiCOLAS VERZELEN ${ }^{\S}$ \\ Université Paris-Ouest*, Université Paris Dauphine ${ }^{\dagger}$, CREST-ENSAE ${ }^{\ddagger}$, and \\ INRA ${ }^{\S}$
}

\begin{abstract}
We consider the problem of estimation of a linear functional in the Gaussian sequence model where the unknown vector $\theta \in \mathbb{R}^{d}$ belongs to a class of $s$-sparse vectors with unknown $s$. We suggest an adaptive estimator achieving a nonasymptotic rate of convergence that differs from the minimax rate at most by a logarithmic factor. We also show that this optimal adaptive rate cannot be improved when $s$ is unknown. Furthermore, we address the issue of simultaneous adaptation to $s$ and to the variance $\sigma^{2}$ of the noise. We suggest an estimator that achieves the optimal adaptive rate when both $s$ and $\sigma^{2}$ are unknown.
\end{abstract}

1. Introduction. We consider the model

$$
y_{j}=\theta_{j}+\sigma \xi_{j}, \quad j=1, \ldots, d,
$$

where $\theta=\left(\theta_{1}, \ldots, \theta_{d}\right) \in \mathbb{R}^{d}$ is an unknown vector of parameters, $\xi_{j}$ are i.i.d. standard normal random variables, and $\sigma>0$ is the noise level. We study the problem of estimation of the linear functional

$$
L(\theta)=\sum_{i=1}^{d} \theta_{i},
$$

based on the observations $y=\left(y_{1}, \ldots, y_{d}\right)$.

For $s \in\{1, \ldots, d\}$, we denote by $\Theta_{s}$ the class of all $\theta \in \mathbb{R}^{d}$ satisfying $\|\theta\|_{0} \leq s$, where $\|\theta\|_{0}$ denotes the number of nonzero components of $\theta$. We assume that $\theta$ belongs to $\Theta_{s}$ for some $s \in\{1, \ldots, d\}$. Parameter $s$ characterizes the sparsity of vector $\theta$. The problem of estimation of $L(\theta)$ in this context arises, for example,

\footnotetext{
Received November 2016; revised October 2017.

${ }^{1}$ The work of O. Collier has been conducted as part of the project Labex MME-DII (ANR11-LBX0023-01).

${ }^{2}$ Supported by GENES and by the French National Research Agency (ANR) under the Grants IPANEMA (ANR-13-BSH1-0004-02) and Labex Ecodec (ANR-11-LABEX-0047). Also supported by the "Chaire Economie et Gestion des Nouvelles Données," under the auspices of Institut Louis Bachelier, Havas-Media and Paris-Dauphine.
}

MSC2010 subject classifications. 62J05, $62 \mathrm{G} 05$.

Key words and phrases. Nonasymptotic minimax estimation, adaptive estimation, linear functional, sparsity, unknown noise variance. 
if one wants to estimate the value of a function $f$ at a fixed point from noisy observations of its Fourier coefficients knowing that the function admits a sparse representation with respect to the first $d$ functions of the Fourier basis. Indeed, in this case the value $f(0)$ is equal to the sum of Fourier coefficients of $f$ with even indices.

As a measure of quality of an estimator $\hat{T}$ of the functional $L(\theta)$ based on the sample $\left(y_{1}, \ldots, y_{d}\right)$, we consider the maximum squared risk

$$
\psi_{s}^{\hat{T}} \triangleq \sup _{\theta \in \Theta_{s}} \mathbf{E}_{\theta}(\hat{T}-L(\theta))^{2}
$$

where $\mathbf{E}_{\theta}$ denotes the expectation with respect to the distribution $\mathbf{P}_{\theta}$ of $\left(y_{1}, \ldots, y_{d}\right)$ satisfying (1). For each fixed $s \in\{1, \ldots, d\}$, the best quality of estimation is characterized by the minimax risk

$$
\psi_{s}^{*} \triangleq \inf _{\hat{T}} \sup _{\theta \in \Theta_{s}} \mathbf{E}_{\theta}(\hat{T}-L(\theta))^{2},
$$

where the infimum is taken over all estimators. An estimator $T^{*}$ is called rate optimal on $\Theta_{s}$ if $\psi_{s}^{T^{*}} \asymp \psi_{s}^{*}$. Here and in the following, we write $a(d, s, \sigma) \asymp$ $b(d, s, \sigma)$ for two functions $a(\cdot)$ and $b(\cdot)$ of $d, s$ and $\sigma$ if there exist absolute constants $c>0$ and $c^{\prime}>0$ such that $c<a(d, s, \sigma) / b(d, s, \sigma)<c^{\prime}$ for all $d$, all $s \in\{1, \ldots, d\}$ and all $\sigma>0$.

The problem of estimation of the linear functional from the minimax point of view has been analyzed in Ibragimov and Khas'minskii (1984), Cai and Low (2004, 2005), Golubev (2004), Golubev and Levit (2004), Laurent, Ludeña and Prieur (2008) among others. Most of these papers study minimax estimation of linear functionals on classes of vectors $\theta$ different from $\Theta_{s}$. Namely, $\theta$ is considered as a vector of first $d$ Fourier or wavelet coefficients of functions belonging to some smoothness class, such as Sobolev or Besov classes. In particular, the class of vectors $\theta$ is assumed to be convex, which is not the case of class $\Theta_{s}$. Cai and Low (2004) were the first to address the problem of constructing rate optimal estimators of $L(\theta)$ on the sparsity class $\Theta_{s}$ and evaluating the minimax risk $\psi_{s}^{*}$. They studied the case $s<d^{a}$ for some $a<1 / 2$, with $\sigma=1 / \sqrt{d}$, and established upper and lower bounds on $\psi_{s}^{*}$ that are accurate up to a logarithmic factor in $d$. The sharp nonasymptotic expression for the minimax risk $\psi_{s}^{*}$ is derived in Collier, Comminges and Tsybakov (2017) where it is shown that, for all $d$, all $s \in\{1, \ldots, d\}$ and all $\sigma>0$

$$
\psi_{s}^{*} \asymp \sigma^{2} s^{2} \log \left(1+d / s^{2}\right) .
$$

Furthermore, Collier, Comminges and Tsybakov (2017) prove that a simple estimator of the form

$$
\hat{L}_{s}^{*}= \begin{cases}\sum_{j=1}^{d} y_{j} \mathbb{1}_{y_{j}^{2}>2 \sigma^{2} \log \left(1+d / s^{2}\right)} & \text { if } s<\sqrt{d}, \\ \sum_{j=1}^{d} y_{j} & \text { otherwise }\end{cases}
$$


is rate optimal. Here and in the following, $\mathbb{1}_{\{.\}}$denotes the indicator function.

Note that the minimax risk $\psi_{s}^{*}$ critically depends on the parameter $s$ that in practice is usually unknown. More importantly, the rate optimal estimator $\hat{L}_{s}^{*}$ depends on $s$ as well, which makes it inaccessible in practice.

In this paper, we suggest adaptive estimators of $L(\theta)$ that do not depend on $s$ and achieve a nonasymptotic rate of convergence $\Phi^{L}(\sigma, s)$ that differs from the minimax rate $\psi_{s}^{*}$ at most by a logarithmic factor. We also show that this rate cannot be improved when $s$ is unknown in the sense of the definition that we give in Section 2 below. Furthermore, in Section 3 we address the issue of simultaneous adaptation to $s$ and $\sigma$. We suggest an estimator that achieves the best rate of adaptive estimation $\Phi^{L}(\sigma, s)$ when both $s$ and $\sigma$ are unknown.

2. Main results. Our aim is to show that the optimal adaptive rate of convergence is of the form

$$
\Phi^{L}(\sigma, s)=\sigma^{2} s^{2} \log \left(1+d(\log d) / s^{2}\right)
$$

and to construct an adaptive estimator attaining this rate. Note that

$$
\Phi^{L}(\sigma, s) \asymp \sigma^{2} d(\log d) \quad \text { for all } \sqrt{d \log d} \leq s \leq d .
$$

Indeed, since the function $x \mapsto x \log (1+1 / x)$ is increasing for $x>0$,

$$
\begin{aligned}
d(\log d) / 2 & \leq s^{2} \log \left(1+d(\log d) / s^{2}\right) \\
& \leq d(\log d) \quad \forall \sqrt{d \log d} \leq s \leq d, d \geq 3 .
\end{aligned}
$$

To construct an adaptive estimator, we first consider a collection of nonadaptive estimators indexed by $s=1, \ldots, d$ :

$$
\hat{L}_{s}= \begin{cases}\sum_{j=1}^{d} y_{j} \mathbb{1}_{y_{j}^{2}>\alpha \sigma^{2} \log \left(1+d(\log d) / s^{2}\right)} & \text { if } s \leq \sqrt{d \log d / 2}, \\ \sum_{j=1}^{d} y_{j} & \text { otherwise, }\end{cases}
$$

where $\alpha>0$ is a constant that will be chosen large enough. Note that if in Definition (5) we replace $d(\log d)$ by $d$, and $\alpha$ by 2 , we obtain the estimator $\hat{L}_{s}^{*}$ suggested in Collier, Comminges and Tsybakov (2017); cf. (2). It is proved in Collier, Comminges and Tsybakov (2017) that the estimator $\hat{L}_{s}^{*}$ is rate optimal in the minimax nonadaptive sense. The additional $\log d$ factor is necessary to achieve adaptivity as it will be clear from the subsequent arguments.

We obtain an adaptive estimator via data-driven selection in the collection of estimators $\left\{\hat{L}_{s}\right\}$. The selection is based on a Lepski type scheme. For $s=1, \ldots, d$, consider the thresholds $\omega_{s}>0$ given by

$$
\omega_{s}^{2}=\beta \sigma^{2} s^{2} \log \left(1+d(\log d) / s^{2}\right)=\beta \Phi^{L}(\sigma, s),
$$


where $\beta>0$ is a constant that will be chosen large enough. We define the selected index $\hat{s}$ by the relation

$$
\hat{s} \triangleq \min \left\{s \in\{1, \ldots,\lfloor\sqrt{d \log d / 2}\rfloor\}:\left|\hat{L}_{s}-\hat{L}_{s^{\prime}}\right| \leq \omega_{s^{\prime}} \text { for all } s^{\prime}>s\right\}
$$

with the convention that $\hat{s}=\lfloor\sqrt{d \log d / 2}\rfloor+1$ if the set in (6) is empty. Here, $\lfloor\sqrt{d \log d / 2}\rfloor$ denotes the largest integer less than $\sqrt{d \log d / 2}$. Finally, we define an adaptive to $s$ estimator of $L$ as

$$
\hat{L} \triangleq \hat{L}_{\hat{s}}
$$

The following theorem exhibits an upper bound on its risk.

THEOREM 1. Assume that $\alpha>48, \beta \geq \frac{16}{9}(\sqrt{12}+2 \sqrt{\alpha})^{2}$ and $d \geq d_{0}$, where $d_{0} \geq 3$ is an absolute constant. Let $\hat{L}$ be the estimator defined in (7). Then, for all $\sigma>0$ and $s \in\{1, \ldots, d\}$, we have

$$
\sup _{\theta \in \Theta_{s}} \mathbf{E}_{\theta}(\hat{L}-L(\theta))^{2} \leq C \Phi^{L}(\sigma, s)
$$

for some absolute constant $C$.

Observe that for small $s$ (such that $s \leq d^{b}$ for $b<1 / 2$ ), we have $1 \leq$ $\Phi^{L}(\sigma, s) / \psi_{s}^{*} \leq c^{\prime}$ where $c^{\prime}>0$ is an absolute constant. Therefore, for such $s$ our estimator $\hat{L}$ attains the best possible rate on $\Theta_{s}$ given by the minimax risk $\psi_{s}^{*}$ and it cannot be improved, even by estimators depending on $s$. Because of this, the only issue is to check that the rate $\Phi^{L}(\sigma, s)$ cannot be improved if $s$ is greater than $d^{b}$ with $b<1 / 2$. For definiteness, we consider below the case $b=1 / 4$ but with minor modifications the argument applies to any $b<1 / 2$. Specifically, we prove that any estimator whose maximal risk over $\Theta_{s}$ is smaller (within a small constant) than $\Phi^{L}(\sigma, s)$ for some $s \geq d^{1 / 4}$, must have a maximal risk over $\Theta_{1}$ of power order in $d$ instead of the logarithmic order $\Phi^{L}(\sigma, 1)$ corresponding to our estimator. In other words, if we find an estimator that improves upon our estimator only slightly (by a constant factor) for some $s \geq d^{1 / 4}$, then this estimator inevitably loses much more for small $s$, such as $s=1$, since there the ratio of maximal risks of the two estimators behaves as a power of $d$.

THEOREM 2. Let $d \geq 6$ and $\sigma>0$. There exist two small absolute constants $C_{0}>0$ and $C_{1}>0$ such that the following holds. Any estimator $\widehat{T}$ that satisfies

$$
\sup _{\theta \in \Theta_{s}} \mathbf{E}_{\theta}\left[(\widehat{T}-L(\theta))^{2}\right] \leq C_{0} \Phi^{L}(\sigma, s) \quad \text { for some } s \geq d^{1 / 4}
$$

has a degenerate maximal risk over $\Theta_{1}$, that is,

$$
\sup _{\theta \in \Theta_{1}} \mathbf{E}_{\theta}\left[(\widehat{T}-L(\theta))^{2}\right] \geq C_{1} \sigma^{2} d^{1 / 4} \text {. }
$$


The property obtained in Theorem 2 can be paraphrased in an asymptotic context to conclude that $\Phi^{L}(\sigma, s)$ is the adaptive rate of convergence on the scale of classes $\left\{\Theta_{s}, s=1, \ldots, d\right\}$ in the sense of the definition in Tsybakov (1998). Indeed, assume that $d \rightarrow \infty$. Following Tsybakov (1998), we call a function $s \mapsto$ $\Psi_{d}(s)$ the adaptive rate of convergence on the scale of classes $\left\{\Theta_{s}, s=1, \ldots, d\right\}$ if the following holds:

(i) There exists an estimator $\hat{L}$ such that, for all $d$,

$$
\max _{s=1, \ldots, d} \sup _{\theta \in \Theta_{s}} \mathbf{E}_{\theta}(\hat{L}-L(\theta))^{2} / \Psi_{d}(s) \leq C,
$$

where $C>0$ is a constant (clearly, such an estimator $\hat{L}$ is adaptive since it cannot depend on $s$ ).

(ii) If there exist another function $s \mapsto \Psi_{d}^{\prime}(s)$ and a constant $C^{\prime}>0$ such that, for all $d$,

$$
\inf _{\hat{T}} \max _{s=1, \ldots, d} \sup _{\theta \in \Theta_{s}} \mathbf{E}_{\theta}(\hat{T}-L(\theta))^{2} / \Psi_{d}^{\prime}(s) \leq C^{\prime},
$$

and

$$
\min _{s=1, \ldots, d} \frac{\Psi_{d}^{\prime}(s)}{\Psi_{d}(s)} \rightarrow 0 \quad \text { as } d \rightarrow \infty,
$$

then there exists $\bar{s} \in\{1, \ldots, d\}$ such that

$$
\frac{\Psi_{d}^{\prime}(\bar{s})}{\Psi_{d}(\bar{s})} \min _{s=1, \ldots, d} \frac{\Psi_{d}^{\prime}(s)}{\Psi_{d}(s)} \rightarrow \infty \quad \text { as } d \rightarrow \infty .
$$

In words, this definition states that the adaptive rate of convergence $\Psi_{d}(s)$ is such that any improvement of this rate for some $s$ [cf. (10)] is possible only at the expense of much greater loss for another $\bar{s}$ [cf. (11)].

COROLLARY 1. The rate $\Phi^{L}(\sigma, s)$ is the adaptive rate of convergence on the scale of classes $\left\{\Theta_{s}, s=1, \ldots, d\right\}$.

It follows from the above results that the rate $\Phi^{L}(\sigma, s)$ cannot be improved when adaptive estimation on the family of sparsity classes $\left\{\Theta_{s}, s=1, \ldots, d\right\}$ is considered. The ratio between the best rate of adaptive estimation $\Phi^{L}(\sigma, s)$ and the minimax rate $\psi_{s}^{*}$ is equal to

$$
\phi_{s}^{*}=\frac{\Phi^{L}(\sigma, s)}{\psi_{s}^{*}}=\frac{\log \left(1+d(\log d) / s^{2}\right)}{\log \left(1+d / s^{2}\right)} .
$$

As mentioned above, $\phi_{s}^{*} \asymp 1$ if $s \leq d^{b}$ for $b<1 / 2$. In a vicinity of $s=\sqrt{d}$, we have $\phi_{s}^{*} \asymp \log \log d$, whereas for $s \geq \sqrt{d \log d}$ the behavior of this ratio is $\log$ arithmic: $\phi_{s}^{*} \asymp \log d$. Thus, there are different regimes and we see that, in some 
of them, rate adaptive estimation of the linear functional on the sparsity classes is impossible without loss of efficiency as compared to the minimax estimation. However, this loss is at most logarithmic in $d$.

We study now the adaptive rate of convergence on restricted scale of classes $\left\{\Theta_{s}, d^{r_{1}} \leq s \leq d^{r_{2}}\right\}$ for some $0<r_{1}<r_{2} \leq 1$.

PROPOSITION 1. Fix $0<r_{1}<r_{2} \leq 1$. The adaptive rate of convergence on the scale of classes $\left\{\Theta_{s}, d^{r_{1}} \leq s \leq d^{r_{2}}\right\}$ is $\Phi^{L}(\sigma, s)$ if $r_{1}<1 / 2$ and $\sigma^{2} d$ if $r_{1} \geq 1 / 2$.

Proof of Proposition 1. For $r_{1} \geq 1 / 2$, it is proved in Collier, Comminges and Tsybakov (2017) that the simple estimator $\widehat{L}_{d}^{*}=\sum_{j=1}^{d} y_{j}$ simultaneously achieves the minimax risk $\psi_{s}^{*} \asymp \sigma^{2} d$ for all $s=\lfloor\sqrt{d}\rfloor, \ldots, d$. As a consequence, there is no loss for adaptation to the classes $\left\{\Theta_{s}, d^{1 / 2} \leq s \leq d\right\}$.

Now assume that $r_{1}<1 / 2$. In view of Theorem 1 , the estimator $\widehat{L}$ simultaneously achieves the rate $\Phi^{L}(\sigma, s)$ for all classes $\left\{\Theta_{s}, d^{r_{1}} \leq s \leq d^{r_{2}}\right\}$. It suffices to prove that this rate is optimal. Below, $\lceil x\rceil$ stands for the smallest integer greater than or equal to $x$.

Proposition 2. Fix $r_{1} \in(1 / 4,1 / 2)$ Let $d \geq 6$ and $\sigma>0$. There exist two absolute constants $C_{0}>0$ and $C_{1}>0$ such that the following holds. Any estimator $\widehat{T}$ that satisfies

$$
\sup _{\theta \in \Theta_{s}} \mathbf{E}_{\theta}\left[(\widehat{T}-L(\theta))^{2}\right] \leq C_{0}\left(1 / 2-r_{1}\right) \Phi^{L}(\sigma, s) \quad \text { for some } s \geq d^{\left(1 / 2+r_{1}\right) / 2}
$$

has a degenerate maximal risk over $\Theta_{\left\lceil d^{r}{ }_{1}\right\rceil}$, that is,

$$
\sup _{\theta \in \Theta_{\left\lceil d^{r} 1\right\rceil}} \mathbf{E}_{\theta}\left[(\widehat{T}-L(\theta))^{2}\right] \geq C_{1}\left(1 / 2-r_{1}\right) \sigma^{2} d^{3 r_{1} / 2+1 / 2} .
$$

Note that $\Phi^{L}\left(\sigma,\left\lceil d^{r_{1}}\right\rceil\right)$ is not of larger order than $\sigma^{2} d^{2 r_{1}} \log (d)$, which is much smaller than $d^{3 r_{1} / 2+1 / 2}$. The proof of Proposition 2 follows immediately by applying Lemma 7 with $a=\left(r_{1}+1 / 2\right) / 2$ and then concluding the proof as in Corollary 1.

3. Adaptation to $s$ when $\sigma$ is unknown. In this section, we discuss a generalization of our adaptive estimator to the case when the standard deviation $\sigma$ of the noise is unknown.

To treat the case of unknown $\sigma$, we first construct an estimator $\hat{\sigma}$ of $\sigma$ such that, with high probability, $\sigma \leq \hat{\sigma} \leq 10 \sigma$. Then we consider the family of estimators defined by a relation analogous to (5):

$$
\hat{L}_{s}^{\prime}= \begin{cases}\sum_{j=1}^{d} y_{j} \mathbb{1}_{y_{j}^{2}>\alpha \hat{\sigma}^{2} \log \left(1+d(\log d) / s^{2}\right)} & \text { if } s \leq \sqrt{d \log d / 2} \\ \sum_{j=1}^{d} y_{j} & \text { otherwise }\end{cases}
$$


where $\alpha>0$ is a constant to be chosen large enough. The difference from (5) consists in the fact that we replace the unknown $\sigma$ by $\hat{\sigma}$. Then we define a random threshold $\omega_{s}^{\prime}>0$ as

$$
\left(\omega_{s}^{\prime}\right)^{2}=\beta \hat{\sigma}^{2} s^{2} \log \left(1+d(\log d) / s^{2}\right),
$$

where $\beta>0$ is a constant to be chosen large enough. The selected index $\hat{s}^{\prime}$ is defined by the formula analogous to (6):

$$
\hat{s}^{\prime} \triangleq \min \left\{s \in\{1, \ldots,\lfloor\sqrt{d \log d / 2}\rfloor\}:\left|\hat{L}_{s}^{\prime}-\hat{L}_{s^{\prime}}^{\prime}\right| \leq \omega_{s^{\prime}}^{\prime} \text { for all } s^{\prime}>s\right\} .
$$

Finally, the adaptive estimator when $\sigma$ is unknown is defined as

$$
\hat{L}^{\prime} \triangleq \hat{L}_{\hat{s}^{\prime}}^{\prime}
$$

The aim of this section is to show that the risk of the estimator $\hat{L}^{\prime}$ admits an upper bound with the same rate as in Theorem 1 for all $d$ large enough. Consequently, $\hat{L}^{\prime}$ attains the best rate of adaptive estimation as follows from Section 2 .

Different estimators $\hat{\sigma}$ can be used. By slightly modifying the method suggested in Collier, Comminges and Tsybakov (2017), we consider the statistic

$$
\hat{\sigma}=9\left(\frac{1}{\lfloor d / 2\rfloor} \sum_{j \leq d / 2} y_{(j)}^{2}\right)^{1 / 2},
$$

where $y_{(1)}^{2} \leq \cdots \leq y_{(d)}^{2}$ are the order statistics associated to $y_{1}^{2}, \ldots, y_{d}^{2}$. This statistic has the properties stated in the next proposition. In particular, $\hat{\sigma}$ overestimates $\sigma$ but it turns out to be without prejudice to the attainment of the best rate by the resulting estimator $\hat{L}_{s}^{\prime}$.

PROPOSITION 3. There exists an absolute constant $d_{0} \geq 3$ such that the following holds. Let $\hat{\sigma}$ be the estimator defined in (14). Then, for all integers $d \geq d_{0}$ and $s<d / 2$ we have

$$
\inf _{\theta \in \Theta_{s}} \mathbf{P}_{\theta}(\sigma \leq \hat{\sigma} \leq 10 \sigma) \geq 1-d^{-5},
$$

and

$$
\sup _{\theta \in \Theta_{s}} \mathbf{E}_{\theta}\left(\hat{\sigma}^{4}\right) \leq \bar{C} \sigma^{4}
$$

where $\bar{C}$ is an absolute constant.

The proof of this proposition is given in Section 4. Using Proposition 3, we establish the following bound on the risk of the estimator $\hat{L}^{\prime}$. 
THEOREM 3. Assume that $\alpha>48, \beta \geq \frac{16}{9}(\sqrt{12}+2 \sqrt{\alpha})^{2}$ and $d \geq d_{0}$ where $d_{0} \geq 3$ is an absolute constant. Let $\hat{\sigma}$ be the estimator defined in (14). Then, for the estimator $\hat{L}^{\prime}$ with tuning parameters $\alpha$ and $\beta$, for all $\sigma>0$, and all $s<d / 2$ we have

$$
\sup _{\theta \in \Theta_{s}} \mathbf{E}_{\theta}\left(\hat{L}^{\prime}-L(\theta)\right)^{2} \leq C \Phi^{L}(\sigma, s)
$$

for some absolute constant $C$.

Thus, the estimator $\hat{L}^{\prime}$, which is independent of both $s$ and $\sigma$ achieves the rate $\Phi^{L}(\sigma, s)$ that is, the best possible rate of adaptive estimation established in Section 2 .

The condition $s<d / 2$ in this theorem can be generalized to $s \leq \zeta d$ for some $\zeta \in(0,1)$. In fact, for any $\zeta \in(0,1)$, we can modify the definition of (14) by summing only over the $(1-\zeta) d$ smallest values of $y_{i}^{2}$. Then, changing the numerical constants $\alpha$ and $\beta$ in the definition of $\omega_{s}^{\prime}$, we obtain that the corresponding estimator $\hat{L}^{\prime}$ achieves the best possible rate simultaneously for all $s \leq \zeta d$ with a constant $C$ in (17) that would depend on $\zeta$. However, we cannot set $\zeta=1$. Indeed, the following proposition shows that it is not possible to construct an estimator, which is simultaneously adaptive to all $\sigma>0$ and to all $s \in[1, d]$.

PROPOSITION 4. Let $d \geq 3$ and $\sigma>0$. There exists a small absolute constant $C_{0}>0$ such that the following holds. Any estimator $\widehat{T}$ that satisfies

$$
\sup _{\theta \in \Theta_{1}} \mathbf{E}_{\theta}\left[(\widehat{T}-L(\theta))^{2}\right] \leq C_{0} \sigma^{2} d \quad \forall \sigma>0,
$$

has a degenerate maximal risk over $\Theta_{d}$, that is, for any fixed $\sigma>0$,

$$
\sup _{\theta \in \Theta_{d}} \mathbf{E}_{\theta}\left[(\widehat{T}-L(\theta))^{2}\right]=\infty \text {. }
$$

In other words, when $\sigma$ is unknown, any estimator, for which the maximal risk over $\Theta_{d}$ is finite for all $\sigma$, cannot achieve over $\Theta_{1}$ a risk of smaller order than $\sigma^{2} d$, and hence cannot be minimax adaptive. Indeed, as shown above, the adaptive minimax rate over $\Theta_{1}$ is of the order $\sigma^{2} \log d$.

4. Proofs of the upper bounds. In the following, we will denote $c_{1}, c_{2}, \ldots$ absolute positive constants and write for brevity $L$ instead of $L(\theta)$.

4.1. Proof of Theorem 1. Let $s \in\{1, \ldots, d\}$ and assume that $\theta$ belongs to $\Theta_{s}$. We have

$$
\mathbf{E}_{\theta}(\hat{L}-L)^{2}=\mathbf{E}_{\theta}\left[\left(\hat{L}_{\hat{s}}-L\right)^{2} \mathbb{1}_{\hat{s} \leq s}\right]+\mathbf{E}_{\theta}\left[\left(\hat{L}_{\hat{s}}-L\right)^{2} \mathbb{1}_{\hat{s}>s}\right]
$$


Consider the first summand on the right-hand side of (20). Set for brevity $s_{0}=$ $\lfloor\sqrt{d \log d / 2}\rfloor+1$. Using the definition of $\hat{s}$ we obtain, on the event $\{\hat{s} \leq s\}$,

$$
\left(\hat{L}_{\hat{s}}-L\right)^{2} \leq 2 \omega_{s}^{2}+2\left(\hat{L}_{s}-L\right)^{2} \quad \text { if } s<s_{0} \text { or } s \geq s_{0}, \hat{s}<s_{0} .
$$

Thus,

$$
\begin{aligned}
& \forall s<s_{0}: \quad \mathbf{E}_{\theta}\left[\left(\hat{L}_{\hat{s}}-L\right)^{2} \mathbb{1}_{\hat{s} \leq s}\right] \leq 2 \beta \Phi^{L}(\sigma, s)+2 \mathbf{E}_{\theta}\left(\hat{L}_{s}-L\right)^{2}, \\
& \forall s \geq s_{0}: \quad \mathbf{E}_{\theta}\left[\left(\hat{L}_{\hat{s}}-L\right)^{2} \mathbb{1}_{\hat{s} \leq s}\right] \leq \mathbf{E}_{\theta}\left[\left(\hat{L}_{\hat{s}}-L\right)^{2}\left(\mathbb{1}_{\hat{s} \leq s, \hat{s}<s_{0}}+\mathbb{1}_{\hat{s}=s_{0}}\right)\right] \\
& \leq 2 \beta \Phi^{L}(\sigma, s)+2 \mathbf{E}_{\theta}\left(\hat{L}_{s}-L\right)^{2} \\
& +\mathbf{E}_{\theta}\left(\hat{L}_{s_{0}}-L\right)^{2} .
\end{aligned}
$$

By Lemma 6 proved at the end of this section, we have

$$
\sup _{\theta \in \Theta_{s}} \mathbf{E}_{\theta}\left(\hat{L}_{s}-L\right)^{2} \leq c_{1} \Phi^{L}(\sigma, s), \quad s=1, \ldots, s_{0}-1 .
$$

Note that, in view of (3), for all $s \in\left[s_{0}, d\right]$ we have

$$
\Phi^{L}\left(\sigma, s_{0}\right) \leq \sigma^{2} d \log d \leq 2 \sigma^{2} s^{2} \log \left(1+(d \log d) / s^{2}\right)=2 \Phi^{L}(\sigma, s),
$$

and by definition of $\hat{L}_{s}$, for all $s \in\left[s_{0}, d\right]$ and all $\theta \in \mathbb{R}^{d}$, we have $\mathbf{E}_{\theta}\left(\hat{L}_{s}-L\right)^{2} \leq$ $\sigma^{2} d \leq 2 \Phi^{L}(\sigma, s)$. Combining these remarks with (21) and (22) yields

$$
\sup _{\theta \in \Theta_{s}} \mathbf{E}_{\theta}\left[\left(\hat{L}_{\hat{s}}-L\right)^{2} \mathbb{1}_{\hat{s} \leq s}\right] \leq c_{2} \Phi^{L}(\sigma, s), \quad s=1, \ldots, d .
$$

Consider now the second summand on the right-hand side of (20). Since $\hat{s} \leq s_{0}$, we obtain the following two facts. First,

$$
\sup _{\theta \in \Theta_{s}} \mathbf{E}_{\theta}\left[\left(\hat{L}_{\hat{s}}-L\right)^{2} \mathbb{1}_{\hat{s}>s}\right]=0 \quad \forall s \geq s_{0} .
$$

Second, on the event $\{\hat{s}>s\}$,

$$
\left(\hat{L}_{\hat{s}}-L\right)^{4} \leq \sum_{s<s^{\prime} \leq s_{0}}\left(\hat{L}_{s^{\prime}}-L\right)^{4} .
$$

Thus,

$$
\begin{aligned}
\sup _{\theta \in \Theta_{s}} & \mathbf{E}_{\theta}\left[\left(\hat{L}_{\hat{s}}-L\right)^{2} \mathbb{1}_{\hat{s}>s}\right] \\
& \leq \sup _{\theta \in \Theta_{s}}\left[\sqrt{\mathbf{P}_{\theta}(\hat{s}>s)}(d \log d)^{1 / 4} \max _{s<s^{\prime} \leq s_{0}} \sqrt{\mathbf{E}_{\theta}\left(\hat{L}_{s^{\prime}}-L\right)^{4}}\right] \\
& \leq(d \log d)^{1 / 4} \sup _{\theta \in \Theta_{s}} \sqrt{\mathbf{P}_{\theta}(\hat{s}>s)} \max _{s^{\prime} \leq s_{0}}\left[\sup _{\theta \in \Theta_{s^{\prime}}} \sqrt{\mathbf{E}_{\theta}\left(\hat{L}_{s^{\prime}}-L\right)^{4}}\right],
\end{aligned}
$$

where for the second inequality we have used that $\Theta_{s} \subset \Theta_{s^{\prime}}$ for $s<s^{\prime}$. To evaluate the right- hand side of (25), we use the following two lemmas. 
LEMMA 1. Recall the definitions of $\hat{L}_{s}$ and $\hat{L}_{s}^{\prime}$ in (5) and (12). For all $s \leq$ $s_{0}=\lfloor\sqrt{d \log d / 2}\rfloor+1$, we have

$$
\begin{aligned}
& \sup _{\theta \in \Theta_{s}} \mathbf{E}_{\theta}\left(\hat{L}_{s}-L\right)^{4} \leq c_{3} \sigma^{4} d^{4}(\log d)^{2}, \\
& \sup _{\theta \in \Theta_{s}} \mathbf{E}_{\theta}\left(\hat{L}_{s}^{\prime}-L\right)^{4} \leq c_{4} \sigma^{4} d^{4}(\log d)^{2} .
\end{aligned}
$$

LEMMA 2. Assume that $\alpha>48$ and $\beta=\frac{16}{9}(\sqrt{12}+2 \sqrt{\alpha})^{2}$.

(i) We have

$$
\max _{s \leq \sqrt{d \log d / 2}} \sup _{\theta \in \Theta_{s}} \mathbf{P}_{\theta}(\hat{s}>s) \leq c_{5} d^{-5} .
$$

(ii) We have

$$
\max _{s \leq \sqrt{d \log d / 2}} \sup _{\theta \in \Theta_{s}} \mathbf{P}_{\theta}\left(\hat{s}^{\prime}>s\right) \leq c_{6} d^{-5}
$$

From (24), (25), the first inequality in Lemma 1, and part (i) of Lemma 2 we find that

$$
\sup _{\theta \in \Theta_{s}} \mathbf{E}_{\theta}\left[\left(\hat{L}_{\hat{s}}-L\right)^{2} \mathbb{1}_{\hat{s}>s}\right] \leq \sqrt{c_{3} c_{5}} \sigma^{2} \leq c_{7} \Phi^{L}(\sigma, s), \quad s=1, \ldots, d .
$$

Combining this inequality with (20) and (23), we obtain the theorem.

\subsection{Proofs of the lemmas.}

Proof of Lemma 1. For $s=s_{0}, \hat{L}_{s}-L=\hat{L}_{s}^{\prime}-L=\sigma \sum_{i=1}^{d} \xi_{i}$. As a consequence,

$$
\mathbf{E}_{\theta}\left(\hat{L}_{s}-L\right)^{4}=\mathbf{E}_{\theta}\left(\hat{L}_{s}^{\prime}-L\right)^{4}=3 \sigma^{4} d^{2} \leq 3 \sigma^{4} d^{4}(\log d)^{2} .
$$

Henceforth, we focus on the case $s \leq \sqrt{d \log (d) / 2}$. We have

$$
\hat{L}_{s}-L=\sigma \sum_{i=1}^{d} \xi_{i}-\sum_{i=1}^{d} y_{i} \mathbb{1}_{y_{i}^{2} \leq \alpha \sigma^{2} \log \left(1+d(\log d) / s^{2}\right)} .
$$

Thus,

$$
\begin{aligned}
\mathbf{E}_{\theta}\left(\hat{L}_{s}-L\right)^{4} & \leq 8\left(\sigma^{4} \mathbf{E}\left(\sum_{i=1}^{d} \xi_{i}\right)^{4}+d^{4} \alpha^{2} \sigma^{4} \log ^{2}\left(1+d(\log d) / s^{2}\right)\right) \\
& \leq c_{3} \sigma^{4} d^{4}(\log d)^{2}
\end{aligned}
$$

In a similar way,

$$
\hat{L}_{s}^{\prime}-L=\sigma \sum_{i=1}^{d} \xi_{i}-\sum_{i=1}^{d} y_{i} \mathbb{1}_{y_{i}^{2} \leq \alpha \hat{\sigma}^{2} \log \left(1+d(\log d) / s^{2}\right)},
$$


and

$$
\mathbf{E}_{\theta}\left(\hat{L}_{s}^{\prime}-L\right)^{4} \leq 8\left(\sigma^{4} \mathbf{E}\left(\sum_{i=1}^{d} \xi_{i}\right)^{4}+d^{4} \alpha^{2} \mathbf{E}_{\theta}\left(\hat{\sigma}^{4}\right) \log ^{2}\left(1+d(\log d) / s^{2}\right)\right) .
$$

The desired bound for $\mathbf{E}_{\theta}\left(\hat{L}_{s}^{\prime}-L\right)^{4}$ follows from this inequality and (16).

ProOF OF LEMma 2. We start by proving part (i) of Lemma 2. Note first that, for $s \leq \sqrt{d \log d / 2}$ and all $\theta$ we have

$$
\mathbf{P}_{\theta}\left(\left|\hat{L}_{s}-L\right|>3 \omega_{s^{\prime}} / 4\right) \leq \mathbf{P}_{\theta}\left(\left|\hat{L}_{s}-L\right|>3 \omega_{s} / 4\right) \quad \forall s<s^{\prime} \leq d .
$$

Indeed, if $s<s^{\prime}$ we have $\omega_{s^{\prime}}>\omega_{s}$ since the function $t \mapsto \omega_{t}$ is increasing for $t>0$. Thus,

$$
\mathbf{P}_{\theta}\left(\left|\hat{L}_{s^{\prime}}-\hat{L}_{s}\right|>\omega_{s^{\prime}}\right) \leq \mathbf{P}_{\theta}\left(\left|\hat{L}_{s^{\prime}}-L\right|>3 \omega_{s} / 4\right)+\mathbf{P}_{\theta}\left(\left|\hat{L}_{s}-L\right|>\omega_{s} / 4\right) .
$$

This inequality and the definition of $\hat{s}$ imply that, for all $s \leq \sqrt{d \log d / 2}$ and all $\theta$,

$$
\begin{aligned}
\mathbf{P}_{\theta}(\hat{s}>s) & \leq \sum_{s<s^{\prime} \leq d} \mathbf{P}_{\theta}\left(\left|\hat{L}_{s^{\prime}}-\hat{L}_{s}\right|>\omega_{s^{\prime}}\right) \\
& \leq d \mathbf{P}_{\theta}\left(\left|\hat{L}_{s}-L\right|>3 \omega_{s} / 4\right)+\sum_{s<s^{\prime} \leq d} \mathbf{P}_{\theta}\left(\left|\hat{L}_{s^{\prime}}-L\right|>\omega_{s^{\prime}} / 4\right)
\end{aligned}
$$

Note that, for $\sqrt{d \log d / 2}<s^{\prime} \leq d$, we have $\hat{L}_{s^{\prime}}=\sum_{i=1}^{d} y_{i}$, and $\omega_{s^{\prime}} \geq \sigma \times$ $\sqrt{\beta d \log d} \sqrt{\log (3) / 2}$ by monotonicity. Hence, for $\sqrt{d \log d / 2}<s^{\prime} \leq d$, and all $\theta$,

$$
\mathbf{P}_{\theta}\left(\left|\hat{L}_{s^{\prime}}-L\right|>\omega_{s^{\prime}} / 4\right) \leq \mathbf{P}\left(\left|\sum_{i=1}^{d} \xi_{i}\right|>\frac{\sqrt{\beta d \log d}}{4} \sqrt{\log (3) / 2}\right) \leq 2 d^{-\beta \log (3) / 64},
$$

where we have used that $\xi_{i}$ are i.i.d. standard Gaussian random variables. This inequality and (30) imply that, for $s \leq \sqrt{d \log d / 2}$, and all $\theta$,

$$
\begin{gathered}
\mathbf{P}_{\theta}(\hat{s}>s) \leq \sqrt{d \log d / 2} \max _{s<s^{\prime} \leq \sqrt{d \log d}} \mathbf{P}_{\theta}\left(\left|\hat{L}_{s^{\prime}}-L\right|>3 \omega_{s^{\prime}} / 4\right) \\
+d \mathbf{P}_{\theta}\left(\left|\hat{L}_{s}-L\right|>3 \omega_{s} / 4\right)+2 d^{-\beta \log (3) / 64}
\end{gathered}
$$

As $\Theta_{s} \subset \Theta_{s^{\prime}}$ for $s<s^{\prime}$, we have

$$
\begin{aligned}
& \max _{s<s^{\prime} \leq \sqrt{d \log d}} \sup _{\theta \in \Theta_{s}} \mathbf{P}_{\theta}\left(\left|\hat{L}_{s^{\prime}}-L\right|>3 \omega_{s^{\prime}} / 4\right) \\
& \leq \max _{s^{\prime} \leq \sqrt{d \log d}} \sup _{\theta \in \Theta_{s^{\prime}}} \mathbf{P}_{\theta}\left(\left|\hat{L}_{s^{\prime}}-L\right|>3 \omega_{s^{\prime}} / 4\right) .
\end{aligned}
$$


Together with (31), this implies

$$
\begin{aligned}
& \max _{s \leq \sqrt{d} \log d} \sup _{\theta \in \Theta_{s}} \mathbf{P}_{\theta}(\hat{s}>s) \\
& \quad \leq 2 d \max _{s^{\prime} \leq \sqrt{d \log d}} \sup _{\theta \in \Theta_{s^{\prime}}} \mathbf{P}_{\theta}\left(\left|\hat{L}_{s^{\prime}}-L\right|>3 \omega_{s^{\prime}} / 4\right)+2 d^{-\beta \log (3) / 64} .
\end{aligned}
$$

Considering the assumption on $\beta$, the last summand in this inequality does not exceed $2 d^{-5}$. Thus, it remains to bound the first term on the right-hand side.

Fix $s \leq \sqrt{d \log d / 2}$ and let $\theta$ belong to $\Theta_{s}$. We will denote by $S$ the support of $\theta$ and we set for brevity

$$
a \triangleq \sqrt{\log \left(1+d(\log d) / s^{2}\right)} .
$$

From (27) and the fact that $y_{i}=\theta_{i}+\sigma \xi_{i}$, we have

$$
\begin{aligned}
\left|\hat{L}_{S}-L\right| & =\left|\sigma \sum_{i \in S} \xi_{i}-\sum_{i \in S} y_{i} \mathbb{1}_{y_{i}^{2} \leq \alpha \sigma^{2} a^{2}}+\sigma \sum_{i \notin S} \xi_{i} \mathbb{1}_{\xi_{i}^{2}>\alpha a^{2}}\right| \\
& \leq \sigma\left|\sum_{i \in S} \xi_{i}\right|+\sigma\left|\sum_{i \notin S} \xi_{i} \mathbb{1}_{\xi_{i}^{2}>\alpha a^{2}}\right|+\sqrt{\alpha} \sigma s a .
\end{aligned}
$$

Recalling that $\omega_{s}=\sqrt{\beta} \sigma s a$ we find

$$
\begin{aligned}
\mathbf{P}_{\theta}\left(\left|\hat{L}_{s}-L\right|>3 \omega_{s} / 4\right) \leq & \mathbf{P}\left(\left|\sum_{i \notin S} \xi_{i} \mathbb{1}_{\xi_{i}^{2}>\alpha a^{2}}\right|>\sqrt{\alpha} s a\right) \\
& +\mathbf{P}\left(\left|\sum_{i \in S} \xi_{i}\right|>(3 \sqrt{\beta} / 4-2 \sqrt{\alpha}) s a\right) .
\end{aligned}
$$

Since $\xi_{i}$ are i.i.d. $\mathcal{N}(0,1)$ random variables, we have

$$
\mathbf{P}\left(\left|\sum_{i \in S} \xi_{i}\right|>(3 \sqrt{\beta} / 4-2 \sqrt{\alpha}) s a\right) \leq 2 \exp \left(-\frac{(3 \sqrt{\beta} / 4-2 \sqrt{\alpha})^{2}}{2} s a^{2}\right) .
$$

We now use the relation

$$
s a^{2}=s \log \left(1+d(\log d) / s^{2}\right) \geq \log d \quad \text { for all } s \in[1, \sqrt{d \log d / 2}],
$$

since the function $s \rightarrow s \log \left(1+d \log (d) / s^{2}\right)$ is increasing. It follows from (34), (35) and the assumption on $\alpha$ and $\beta$ that

$$
\mathbf{P}\left(\left|\sum_{i \in S} \xi_{i}\right|>(3 \sqrt{\beta} / 4-2 \sqrt{\alpha}) s a\right) \leq 2 d^{-6} .
$$

Next, consider the first probability on the right-hand side of (33). To bound it from above, we invoke the following lemma. 
LEMMA 3. If $\alpha>48$, for all $s \leq \sqrt{d \log d / 2}$ and all $U \subseteq\{1, \ldots, d\}$,

$$
\mathbf{P}\left(\sup _{t \in[1,10]}\left|\sum_{i \in U} \xi_{i} \mathbb{1}_{\left|\xi_{i}\right|>\sqrt{\alpha} a t}\right|>\sqrt{\alpha} s a\right) \leq c_{8} d^{-6} \text {. }
$$

Combining (33), (36) and Lemma 3, we obtain part (i) of Lemma 2.

We now proceed to the proof of part (ii) of Lemma 2. Proposition 3 implies that, for $s \leq \sqrt{d \log d}$ and $\theta \in \Theta_{s}$,

$$
\mathbf{P}_{\theta}\left(\hat{s}^{\prime}>s\right) \leq \mathbf{P}_{\theta}\left(\hat{s}^{\prime}>s, \hat{\sigma} \in[\sigma, 10 \sigma]\right)+\mathbf{P}_{\theta}(\hat{\sigma} \notin[\sigma, 10 \sigma]) .
$$

On the event $\{\hat{\sigma} \in[\sigma, 10 \sigma]\}$, we can replace $\hat{\sigma}$ in the definition of $\hat{s}^{\prime}$ either by $\sigma$ or by $10 \sigma$ according to cases, thus making the analysis of $\mathbf{P}_{\theta}\left(\hat{s}^{\prime}>s, \hat{\sigma} \in[\sigma, 10 \sigma]\right)$ equivalent, up to the values of numerical constants, to the analysis of $\mathbf{P}_{\theta}(\hat{s}>s)$ given below. The only nontrivial difference consists in the fact that the analog of (32) when $\hat{L}_{S}$ is replaced by $\hat{L}_{s}^{\prime}$ contains the term $\sigma\left|\sum_{i \notin S} \xi_{i} \mathbb{1}_{\xi_{i}^{2}>\alpha \hat{\sigma}^{2} a^{2} / \sigma^{2}}\right|$ instead of $\sigma\left|\sum_{i \notin S} \xi_{i} \mathbb{1}_{\xi_{i}^{2}>\alpha a^{2}}\right|$ while $\hat{\sigma}$ depends on $\xi_{1}, \ldots, \xi_{d}$. This term is evaluated using Lemma 3 and the fact that

$$
\begin{array}{r}
\mathbf{P}\left(\left|\sum_{i \notin S} \xi_{i} \mathbb{1}_{\left|\xi_{i}\right|>\sqrt{\alpha} \hat{\sigma} a / \sigma}\right|>\sqrt{\alpha} s a, \hat{\sigma} \in[\sigma, 10 \sigma]\right) \\
\leq \mathbf{P}\left(\sup _{t \in[1,10]}\left|\sum_{i \notin S} \xi_{i} \mathbb{1}_{\left|\xi_{i}\right|>\sqrt{\alpha} a t \mid}\right|>\sqrt{\alpha} s a\right) .
\end{array}
$$

We omit further details that are straightforward from inspection of the proof of part (i) of Lemma 2 given above. Thus, part (ii) of Lemma 2 follows.

For the proof of Lemma 3, recall the following fact about the tails of the standard Gaussian distribution, which can be proven by integration by part.

Lemma 4. Let $X \sim \mathcal{N}(0,1), x>1$ and $q \in \mathbb{N}$. There is a constant $C_{q}^{*}$ such that

$$
\mathbf{E}\left[X^{2 q} \mathbb{1}_{|X|>x}\right] \leq C_{q}^{*} x^{2 q-1} e^{-x^{2} / 2} .
$$

Moreover, simulations suggest that $C_{1}^{*} \leq 1.1$.

We will also use the Fuk-Nagaev inequality [Petrov (1995), page 78] that we state here for the reader's convenience.

LEMMA 5 (Fuk-Nagaev inequality). Let $p>2$ and $v>0$. Assume that $X_{1}, \ldots, X_{n}$ are independent random variables with $\mathbf{E}\left(X_{i}\right)=0$ and $\mathbf{E}\left|X_{i}\right|^{p}<\infty$, $i=1, \ldots, n$. Then

$$
\mathbf{P}\left(\sum_{i=1}^{n} X_{i}>v\right) \leq(1+2 / p)^{p} \sum_{i=1}^{n} \mathbf{E}\left|X_{i}\right|^{p} v^{-p}+\exp \left(-\frac{2 v^{2}}{(p+2)^{2} e^{p} \sum_{i=1}^{n} \mathbf{E} X_{i}^{2}}\right) .
$$


Proof of Lemma 3. We have

$$
\begin{aligned}
p_{0} & \triangleq \mathbf{P}\left(\sup _{t \in[1,10]}\left|\sum_{i \in U} \xi_{i} \mathbb{1}_{\left|\xi_{i}\right|>\sqrt{\alpha} a t}\right|>\sqrt{\alpha} s a\right) \\
& =\mathbf{E}\left[\mathbf{P}\left(\sup _{t \in[1,10]}\left|\sum_{i \in U} \varepsilon_{i}\right| \xi_{i}\left|\mathbb{1}_{\left|\xi_{i}\right|>\sqrt{\alpha} a t}\right|>\sqrt{\alpha} s a|| \xi_{i} \mid, i \in U\right)\right],
\end{aligned}
$$

where $\varepsilon_{i}$ denotes the sign of $\xi_{i}$. Consider the function

$$
g(x)=\sup _{t \in[1,10]}\left|\sum_{i \in U} x_{i}\right| \xi_{i}\left|\mathbb{1}_{\left|\xi_{i}\right|>\sqrt{\alpha} a t}\right|,
$$

where $x=\left(x_{i}, i \in U\right)$ with $x_{i} \in\{-1,1\}$. For any $i_{0} \in U$, let $g_{i_{0}, u}(x)$ denote the value of this function when we replace $x_{i_{0}}$ by $u \in\{-1,1\}$. Note that, for any fixed $\left(\left|\xi_{i}\right|, i \in U\right)$, we have the bounded differences condition:

$$
\sup _{x}\left|g(x)-g_{i_{0}, u}(x)\right| \leq 2\left|\xi_{i}\right| \mathbb{1}_{\left|\xi_{i}\right|>\sqrt{\alpha} a} \triangleq 2 Z_{i} \quad \forall u \in\{-1,1\}, i_{0} \in U .
$$

The vector of Rademacher random variables $\left(\varepsilon_{1}, \ldots, \varepsilon_{d}\right)$ is independent from $\left(\left|\xi_{1}\right|, \ldots,\left|\xi_{d}\right|\right)$.Thus, for any fixed $\left(\left|\xi_{i}\right|, i \in U\right)$ we can use the bounded differences inequality, which yields

$$
\begin{aligned}
p_{0} & \leq \mathbf{E}\left[\exp \left(-\frac{\alpha s^{2} a^{2}}{2 \sum_{i \in U} Z_{i}^{2}}\right)\right] \\
& \leq \exp \left(-\frac{\alpha s^{2} a^{2}}{2 \Delta}\right)+\mathbf{P}\left(\sum_{i \in U} Z_{i}^{2}>\Delta\right) \quad \forall \Delta>0 .
\end{aligned}
$$

We now set $\Delta=\sum_{i \in U} \mathbf{E} Z_{i}^{2}+d \alpha a^{2} \exp \left(-\alpha a^{2} /(2 p)\right)$ with $p=\alpha / 8>6$.

To bound from above the probability $\mathbf{P}\left(\sum_{i \in U} Z_{i}^{2}>\Delta\right)$, we apply Lemma 5 with $X_{i}=Z_{i}^{2}-\mathbf{E}\left(Z_{i}^{2}\right)$ and $v=\alpha a^{2} d \exp \left(-\alpha a^{2} /(2 p)\right)$. The random variables $X_{i}$ are centered and satisfy, in view of Lemma 4 ,

$$
\mathbf{E}\left|X_{i}\right|^{p} \leq 2^{p-1} \mathbf{E}\left|Z_{i}\right|^{2 p} \leq 2^{p-1} C_{p}^{*}(\sqrt{\alpha} a)^{2 p-1} e^{-\alpha a^{2} / 2} .
$$

Thus, Lemma 5 yields

$$
\begin{aligned}
\mathbf{P}\left(\sum_{i \in U} Z_{i}^{2}>\Delta\right) \leq & C_{p}^{*} 2^{p-1}(1+2 / p)^{p} \frac{(\sqrt{\alpha} a)^{-1}}{d^{p}} \\
& +\exp \left(-\frac{\sqrt{\alpha} a d \exp \left(\alpha a^{2}(1 / 2-1 / p)\right)}{2(p+2)^{2} e^{p} C_{2}^{*}}\right) .
\end{aligned}
$$

The expression in the last display can be made smaller than $c_{9} d^{-6}$ for all $d \geq 3$. 
Finally, using (38) we find

$$
\begin{aligned}
\frac{\alpha s^{2} a^{2}}{2 \Delta} & \geq \frac{\alpha s^{2} a^{2}}{2 d\left(C_{1}^{*} \sqrt{\alpha} a \exp \left(-\alpha a^{2} / 2\right)+\alpha a^{2} \exp \left(-\alpha a^{2} /(2 p)\right)\right)} \\
& \geq \frac{s^{2} \exp \left(\alpha a^{2} /(2 p)\right)}{4.4 d},
\end{aligned}
$$

whereas

$$
\begin{aligned}
\frac{s^{2} \exp \left(\alpha a^{2} /(2 p)\right)}{d} & =\frac{s^{2}}{d}\left(1+\frac{d \log d}{s^{2}}\right)^{\alpha /(2 p)} \\
& =\log d\left(\frac{s^{2}}{d \log (d)}+1\right) \times\left(1+\frac{d \log d}{s^{2}}\right)^{\alpha /(2 p)-1} \\
& \geq 3^{3} \log d
\end{aligned}
$$

for any $s \leq \sqrt{d \log d / 2}$, since $\alpha=8 p$. Hence, for such $s$,

$$
\exp \left(-\frac{\alpha s^{2} a^{2}}{2 \Delta}\right) \leq c_{10} d^{-6}
$$

Thus, Lemma 3 follows.

LEMMA 6. There exists an absolute constant $d_{0} \geq 3$ such that if $\alpha>48$, we have

$$
\begin{aligned}
& \sup _{\theta \in \Theta_{s}} \mathbf{E}_{\theta}\left(\hat{L}_{s}-L\right)^{2} \leq c_{1} \Phi^{L}(\sigma, s), \\
& \sup _{\theta \in \Theta_{s}} \mathbf{E}_{\theta}\left(\hat{L}_{s}^{\prime}-L\right)^{2} \leq c_{11} \Phi^{L}(\sigma, s) \quad \forall s \leq \sqrt{d \log d / 2} .
\end{aligned}
$$

PROOF. We easily deduce from (32) that

$$
\mathbf{E}_{\theta}\left(\hat{L}_{s}-L\right)^{2} \leq 3 \sigma^{2}\left(s+d \mathbf{E}\left[X^{2} \mathbb{1}_{X^{2}>\alpha a^{2}}\right]+\alpha s^{2} a^{2}\right),
$$

where $X \sim \mathcal{N}(0,1)$. By Lemma 4 ,

$$
d \mathbf{E}\left[X^{2} \mathbb{1}_{X^{2}>2 a^{2}}\right] \leq C_{1}^{*} a d \exp \left(-a^{2}\right)=\frac{C_{1}^{*} a d s^{2}}{s^{2}+d \log d} \leq \frac{C_{1}^{*} s^{2} a}{\log d},
$$

which implies that the desired bound for $\mathbf{E}_{\theta}\left(\hat{L}_{s}-L\right)^{2}$ holds since $\alpha \geq 2$. Next, we prove the bound of the lemma for $\mathbf{E}_{\theta}\left(\hat{L}_{s}^{\prime}-L\right)^{2}$. Similarly to (32),

$$
\hat{L}_{s}^{\prime}-L=\sigma \sum_{i \in S} \xi_{i}-\sum_{i \in S} y_{i} \mathbb{1}_{y_{i}^{2} \leq \alpha \hat{\sigma}^{2} a^{2}}+\sigma \sum_{i \notin S} \xi_{i} \mathbb{1}_{\sigma^{2} \xi_{i}^{2}>\alpha \hat{\sigma}^{2} a^{2}} .
$$


This implies

$$
\begin{aligned}
\mathbf{E}_{\theta}\left[\left(\hat{L}_{s}^{\prime}-L\right)^{2} \mathbb{1}_{\hat{\sigma} \in[\sigma, 10 \sigma]}\right] & \leq \mathbf{E}_{\theta}\left(\sigma\left|\sum_{i \in S} \xi_{i}\right|+\sqrt{\alpha} \hat{\sigma} s a+\sigma W\right)^{2} \\
& \leq 3\left(\sigma^{2} s+\alpha \mathbf{E}_{\theta}\left(\hat{\sigma}^{2}\right) a^{2} s^{2}+\sigma^{2} \mathbf{E}\left(W^{2}\right)\right),
\end{aligned}
$$

where $W \triangleq \sup _{t \in[1,10]}\left|\sum_{i \notin S} \xi_{i} \mathbb{1}_{\left|\xi_{i}\right|>\sqrt{\alpha} a t}\right|$. Using Lemma 3 we find that, for all $\alpha>48$,

$$
\begin{aligned}
\mathbf{E}\left(W^{2}\right) & \leq(\sqrt{\alpha} s a)^{2}+\mathbf{E}\left(\sum_{i \notin S}\left|\xi_{i}\right|\right)^{2} \mathbb{1}_{W>\sqrt{\alpha} s a} \\
& \leq \alpha s^{2} a^{2}+\left[\mathbf{E}\left(\sum_{i \notin S}\left|\xi_{i}\right|\right)^{4}\right]^{1 / 2} c_{9} d^{-3} \leq \alpha s^{2} a^{2}+c_{9} \sqrt{3} d^{-1} .
\end{aligned}
$$

Plugging this bound in (39) and using (16), we get

$$
\mathbf{E}_{\theta}\left[\left(\hat{L}_{s}^{\prime}-L\right)^{2} \mathbb{1}_{\hat{\sigma} \in[\sigma, 10 \sigma]}\right] \leq c_{12} \Phi^{L}(\sigma, s) .
$$

On the other hand, by virtue of Lemma 1 and (15),

$$
\begin{aligned}
\mathbf{E}_{\theta}\left[\left(\hat{L}_{s}^{\prime}-L\right)^{2} \mathbb{1}_{\hat{\sigma} \notin[\sigma, 10 \sigma]}\right] & \leq \sqrt{\mathbf{P}_{\theta}(\hat{\sigma} \notin[\sigma, 10 \sigma])} \sqrt{\mathbf{E}_{\theta}\left(\hat{L}_{s}^{\prime}-L\right)^{4}} \\
& \leq \frac{\sqrt{c_{3}} \sigma^{2} \log d}{d^{1 / 2}} \leq c_{13} \Phi^{L}(\sigma, s) .
\end{aligned}
$$

The desired bound for $\mathbf{E}_{\theta}\left(\hat{L}_{s}^{\prime}-L\right)^{2}$ follows from the last two displays.

\subsection{Proofs of Proposition 3 and of Theorem 3.}

Proof of Proposition 3. Since $s \leq d / 2$, there exists a subset $T$ of size $\lfloor d / 2\rfloor$ such that $T \cap S=\varnothing$. By Definition of $\hat{\sigma}^{2}$, we obtain that

$$
\hat{\sigma}^{2} \leq \frac{81 \sigma^{2}}{\lfloor d / 2\rfloor} \sum_{i \in T} \xi_{i}^{2}
$$

This immediately implies (16). To prove (15), note that the Gaussian concentration inequality [cf. Ledoux and Talagrand (1991)] yields

$$
\mathbf{P}\left(\left(\sum_{i \in T} \xi_{i}^{2}\right)^{1 / 2}>\sqrt{100\lfloor d / 2\rfloor / 81}\right) \leq \exp (-c d),
$$

for a positive constant $c$. Therefore,

$$
\mathbf{P}_{\theta}(\hat{\sigma} \leq 10 \sigma) \geq 1-\exp (-c d) .
$$

Next, let $\mathcal{G}$ be the collection of all subsets of $\{1, \ldots, d\}$ of cardinality $\lfloor d / 2\rfloor$. We now establish a bound on the deviations of random variables $Z_{G}=\frac{1}{\sigma^{2}} \sum_{i \in G} y_{i}^{2}$ 
uniformly over all $G \in \mathcal{G}$. Fix any $G \in \mathcal{G}$. The random variable $Z_{G}$ has a chisquare distribution with $\lfloor d / 2\rfloor$ degrees of freedom and noncentrality parameter $\sum_{i \in G} \theta_{i}^{2}$. In particular, this distribution is stochastically larger than a central chisquare distribution with $d^{\prime}=\lfloor d / 2\rfloor$ degrees of freedom. Let $Z$ be a random variable with this central chi-square distribution. For the tail probability of $Z$, we can use Lemma 11.1 in Verzelen (2012) that gives

$$
\mathbf{P}\left(Z \leq \frac{d^{\prime}}{e} x^{2 / d^{\prime}}\right) \leq x \quad \forall x>0 .
$$

Take $x=\left(\begin{array}{c}d \\ d^{\prime}\end{array}\right)^{-1} e^{-d^{\prime} / 2}$. Using the bound $\log \left(\begin{array}{c}d \\ d^{\prime}\end{array}\right) \leq d^{\prime} \log \left(e d / d^{\prime}\right)$, it follows that $\log (1 / x) \leq d^{\prime}\left(\frac{3}{2}+\log \left(\frac{d}{d^{\prime}}\right)\right) \leq d^{\prime}\left(\frac{3}{2}+\log 2\right)+1$. Taking the union bound over all $G \in \mathcal{G}$, we conclude that

$$
\mathbf{P}\left(\inf _{G \in \mathcal{G}} Z_{G} \leq \frac{d^{\prime}}{4 e^{3}}\left(1-\frac{2}{d^{\prime}}\right)\right) \leq e^{-d^{\prime} / 2}<d^{-5} / 2
$$

for all $d$ large enough. Since $\widehat{\sigma}^{2}=\sigma^{2} \frac{81}{d^{\prime}} \inf _{G \in \mathcal{G}} Z_{G}^{2}$, we obtain that $\widehat{\sigma}^{2} \geq \sigma^{2}$ with probability at least $1-d^{-5} / 2$ for all $d$ large enough. Combining this with (40), we get (15) for all $d$ large enough.

Proof of TheOREM 3. We repeat the proof of Theorem 1 replacing there $\hat{L}_{s}$ by $\hat{L}_{s}^{\prime}$ and $\hat{s}$ by $\hat{s}^{\prime}$. The difference is that, in view of (16), the relation (21) now holds with $c_{14} \beta \Phi^{L}(\sigma, s)$ instead of $\beta \Phi^{L}(\sigma, s)$, and we use the results of Lemmas 1,2 and 6 related to $\hat{L}_{s}^{\prime}$ rather than to $\hat{L}_{s}$.

\section{Proofs of the lower bounds.}

5.1. Proof of Theorem 2. Theorem 2 is an immediate consequence of the following lemma with $a=1 / 4$.

LEMMA 7. For all $d \geq 6, a \in[1 / 4,1 / 2)$, and $s \geq d^{a}$,

$$
\begin{aligned}
R(s) & \triangleq \inf _{\tilde{L}}\left\{\mathbf{E}_{0}(\tilde{L}-L)^{2} \sigma^{-2} d^{-3 a+1 / 2}+\sup _{\theta \in \Theta_{s}} \mathbf{E}_{\theta}(\tilde{L}-L)^{2}\left(\Phi^{L}(\sigma, s)\right)^{-1}\right\} \\
& \geq \frac{1 / 2-a}{40} .
\end{aligned}
$$

ProOF. We first introduce some notation. For a probability measure $\mu$ on $\Theta_{s}$, we denote by $\mathbb{P}_{\mu}$ the mixture probability measure $\mathbb{P}_{\mu}=\int_{\Theta_{s}} \mathbf{P}_{\theta} \mu(d \theta)$. Let $\mathcal{S}(s, d)$ denote the set of all subsets of $\{1, \ldots, d\}$ of size $s$, and let $S$ be a setvalued random variable uniformly distributed on $\mathcal{S}(s, d)$. For any $\rho>0$, denote by $\mu_{\rho}$ the distribution of the random variable $\sigma \rho \sum_{j \in S} e_{j}$ where $e_{j}$ is the $j$ th canonical basis vector in $\mathbb{R}^{d}$. Next, let $\chi^{2}(Q, P)=\int(d Q / d P)^{2} d P-1$ denote 
the chi-square divergence between two probability measures $Q$ and $P$ such that $Q \ll P$, and $\chi^{2}(Q, P)=+\infty$ if $Q \ll P$.

Take any $a \in[1 / 4,1 / 2)$ and $s \geq d^{a}$. Set

$$
\rho \triangleq \sqrt{(1 / 2-a) \log \left(1+d(\log d) / s^{2}\right)}=\sqrt{1 / 2-a}\left(\Phi^{L}(\sigma, s)\right)^{1 / 2} /(s \sigma) .
$$

Consider the mixture distribution $\mathbb{P}_{\mu_{\rho}}$ with this value of $\rho$. For any estimator $\tilde{L}$, we have $\sup _{\theta \in \Theta_{s}} \mathbf{E}_{\theta}(\tilde{L}-L)^{2} \geq \mathbb{E}_{\mu_{\rho}}(\tilde{L}-L)^{2} \geq \mathbb{E}_{\mu_{\rho}}\left(\tilde{L}-\mathbb{E}_{\mu_{\rho}}(L)\right)^{2}=\mathbb{E}_{\mu_{\rho}}(\tilde{L}-\sigma s \rho)^{2}$. Therefore,

$$
\begin{aligned}
R(s) \geq & \inf _{\tilde{L}}\left\{\mathbf{E}_{0}\left(\tilde{L}^{2}\right) \sigma^{-2} d^{-3 a+1 / 2}+\mathbb{E}_{\mu_{\rho}}(\tilde{L}-\sigma s \rho)^{2}\left(\Phi^{L}(\sigma, s)\right)^{-1}\right\} \\
\geq & \frac{1 / 2-a}{4} \inf _{\tilde{L}}\left\{\mathbf{P}_{0}(\tilde{L}>\sigma s \rho / 2) \sigma^{-2} d^{-3 a+1 / 2} \Phi^{L}(\sigma, s)\right. \\
& \left.+\mathbb{P}_{\mu_{\rho}}(\tilde{L}<\sigma s \rho / 2)\right\} \\
\geq & \frac{1 / 2-a}{4} \inf _{\mathcal{A}}\left\{\mathbf{P}_{0}(\mathcal{A}) \sigma^{-2} d^{-3 a+1 / 2} \Phi^{L}(\sigma, s)+\mathbb{P}_{\mu_{\rho}}\left(\mathcal{A}^{c}\right)\right\},
\end{aligned}
$$

where $\inf _{\mathcal{A}}$ denotes the infimum over all measurable events $\mathcal{A}$, and $\mathcal{A}^{c}$ denotes the complement of $\mathcal{A}$. It remains to prove that the expression in (42) is not smaller than $(1 / 2-a) / 40$. This will be deduced from the following lemma, the proof of which is given at the end of this section.

LEMMA 8. Let $P$ and $Q$ be two probability measures on a measurable space $(X, \mathcal{U})$. Then, for any $q>0$,

$$
\inf _{\mathcal{A} \in \mathcal{U}}\left\{P(\mathcal{A}) q+Q\left(\mathcal{A}^{c}\right)\right\} \geq \max _{0<\tau<1}\left[\frac{q \tau}{1+q \tau}\left(1-\tau\left(\chi^{2}(Q, P)+1\right)\right)\right] .
$$

We now apply Lemma 8 with $P=\mathbf{P}_{0}, Q=\mathbb{P}_{\mu_{\rho}}$, and

$$
q=\sigma^{-2} d^{-3 a+1 / 2} \Phi^{L}(\sigma, s)=s^{2} d^{-3 a+1 / 2} \log \left(1+\frac{d(\log d)}{s^{2}}\right) .
$$

By Lemma 1 in Collier, Comminges and Tsybakov (2017), the chi-square divergence $\chi^{2}\left(\mathbb{P}_{\mu_{\rho}}, \mathbf{P}_{0}\right)$ satisfies

$$
\chi^{2}\left(\mathbb{P}_{\mu_{\rho}}, \mathbf{P}_{0}\right) \leq\left(1-\frac{s}{d}+\frac{s}{d} e^{\rho^{2}}\right)^{s}-1 \leq\left(1+\frac{s}{d}\left(e^{\rho^{2}}-1\right)\right)^{s} .
$$

Since $\rho^{2}=(1 / 2-a) \log \left(1+\frac{d(\log d)}{s^{2}}\right)$, we find

$$
\begin{aligned}
\chi^{2}\left(\mathbb{P}_{\mu_{\rho}}, \mathbf{P}_{0}\right) & \leq \exp \left[s \log \left[1+\frac{s}{d}\left(\left(1+\frac{d(\log d)}{s^{2}}\right)^{1 / 2-a}-1\right)\right]\right] \\
& \leq \exp \left[s \log \left(1+(1 / 2-a) \frac{\log d}{s}\right)\right] \leq d^{1 / 2-a},
\end{aligned}
$$


where we have used that $(1+x)^{1 / 2-a} \leq 1+(1 / 2-a) x$ for $x>0$. Take

$$
\tau=\left(d^{1 / 2-a}+1\right)^{-1} / 2 .
$$

Then, using (43) and the inequality $s \geq d^{a}$ we find

$$
\begin{aligned}
q \tau & =\frac{s^{2} \log \left(1+\frac{d(\log d)}{s^{2}}\right)}{2 d^{3 a-1 / 2}\left(d^{1 / 2-a}+1\right)} \geq \frac{d^{2 a} \log \left(1+d^{1-2 a}(\log d)\right)}{2 d^{3 a-1 / 2}\left(d^{1 / 2-a}+1\right)} \\
& >\frac{1}{4} \quad \forall d \geq 6 .
\end{aligned}
$$

Lemma 8 and inequalities (44)-(46) imply

$$
\inf _{\mathcal{A}}\left\{\mathbf{P}_{0}(\mathcal{A}) \sigma^{-2} d^{-3 a+1 / 2} \Phi^{L}(\sigma, s)+\mathbb{P}_{\mu_{\rho}}\left(\mathcal{A}^{c}\right)\right\} \geq \frac{q \tau}{2(1+q \tau)} \geq \frac{1}{10} .
$$

Proof of Lemma 8. We follow the same lines as in the proof of Proposition 2.4 in Tsybakov (2009). Thus, for any $\tau \in(0,1)$,

$$
P(\mathcal{A}) \geq \tau(Q(\mathcal{A})-v) \quad \text { where } v=Q\left(\frac{d P}{d Q}<\tau\right) \leq \tau\left(\chi^{2}(Q, P)+1\right) .
$$

Then

$$
\begin{aligned}
\inf _{\mathcal{A}}\left\{P(\mathcal{A}) q+Q\left(\mathcal{A}^{c}\right)\right\} & \geq \inf _{\mathcal{A}}\left\{q \tau(Q(\mathcal{A})-v)+Q\left(\mathcal{A}^{c}\right)\right\} \\
& \geq \min _{0 \leq t \leq 1} \max (q \tau(t-v), 1-t)=\frac{q \tau(1-v)}{1+q \tau} .
\end{aligned}
$$

5.2. Proof of Corollary 1. First, note that condition (8) with $\Psi_{d}(s)=$ $C \Phi^{L}(\sigma, s)$ is satisfied due to Theorem 1. Next, the minimum in condition (10) with $\Psi_{d}(s)=C \Phi^{L}(\sigma, s)$ can be only attained for $s \geq d^{1 / 4}$, since for $s<d^{1 / 4}$ we have $\Phi^{L}(\sigma, s) \asymp \psi_{s}^{*}$ where $\psi_{s}^{*}$ is the minimax rate on $\Theta_{s}$. Thus, it is not possible to achieve a faster rate than $\Phi^{L}(\sigma, s)$ for $s<d^{1 / 4}$ and, therefore, (10) is equivalent to the condition

$$
\min _{s \geq d^{1 / 4}} \frac{\Psi_{d}^{\prime}(s)}{\Phi^{L}(\sigma, s)} \rightarrow 0
$$

and

$$
\min _{s=1, \ldots, d} \frac{\Psi_{d}^{\prime}(s)}{\Phi^{L}(\sigma, s)} \asymp \min _{s \geq d^{1 / 4}} \frac{\Psi_{d}^{\prime}(s)}{\Phi^{L}(\sigma, s)} .
$$

Obviously, $\Psi_{d}^{\prime}(s)$ cannot be of smaller order than the minimax rate $\psi_{s}^{*}$, which implies that

$$
\min _{s \geq d^{1 / 4}} \frac{\Psi_{d}^{\prime}(s)}{\Phi^{L}(\sigma, s)} \geq \min _{s \geq d^{1 / 4}} \frac{c \psi_{s}^{*}}{\Phi^{L}(\sigma, s)}=\min _{s \geq d^{1 / 4}} \frac{c \log \left(1+d / s^{2}\right)}{\log \left(1+d(\log d) / s^{2}\right)} \geq \frac{c^{\prime}}{\log d},
$$


where $c, c^{\prime}>0$ are absolute constants. On the other hand, Theorem 2 yields

$$
\frac{C^{\prime} \Psi_{d}^{\prime}(1)}{\Phi^{L}(\sigma, 1)} \geq \frac{C^{\prime} C_{1} \sigma^{2} d^{1 / 4}}{\Phi^{L}(\sigma, 1)}=\frac{C^{\prime} C_{1} d^{1 / 4}}{\log (1+d(\log d))} .
$$

Combining the last three displays, we find

$$
\frac{\Psi_{d}^{\prime}(1)}{\Phi^{L}(\sigma, 1)} \min _{s=1, \ldots, d} \frac{\Psi_{d}^{\prime}(s)}{\Phi^{L}(\sigma, s)} \geq \frac{c^{\prime} C^{\prime} C_{1} d^{1 / 4}}{(\log d) \log (1+d(\log d))} \rightarrow \infty
$$

as $d \rightarrow \infty$, thus proving (11) with $\bar{s}=1$.

5.3. Proof of Proposition 4. Since in this proof we consider different values of $\sigma$, we denote the probability distribution of $\left(y_{1}, \ldots, y_{d}\right)$ satisfying (1) by $\mathbf{P}_{\theta, \sigma^{2}}$. Let $\mathbf{E}_{\theta, \sigma^{2}}$ be the corresponding expectation. Assume that $\widehat{T}$ satisfies (18) with $C_{0}=1 / 512$. We will prove that (19) holds for $\sigma=1$. The extension to arbitrary $\sigma>0$ is straightforward and is therefore omitted.

Let $a>1$ be a positive number and let $\mu$ be the $d$-dimensional normal distribution with zero mean and covariance matrix $a^{2} \mathbf{I}_{d}$ where $\mathbf{I}_{d}$ is the identity matrix. In what follows, we consider the mixture probability measure $\mathbb{P}_{\mu}=\int_{\Theta_{d}} \mathbf{P}_{\theta, 1} \mu(d \theta)$. Observe that $\mathbb{P}_{\mu}=\mathbf{P}_{0,1+a^{2}}$.

Fixing $\theta=0$ and $\sigma^{2}=1+a^{2}$ in (18), we get $\mathbf{E}_{0,1+a^{2}}\left[\widehat{T}^{2}\right] \leq 2 C_{0} a^{2} d$ and, therefore, $\mathbf{P}_{0,1+a^{2}}\left(|\widehat{T}| \geq \frac{1}{8} a \sqrt{d}\right) \leq \frac{1}{4}$. Since $\mathbb{P}_{\mu}=\mathbf{P}_{0,1+a^{2}}$, this implies

$$
\mathbb{P}_{\mu}\left(|\widehat{T}|<\frac{1}{8} a \sqrt{d}\right)>\frac{3}{4} .
$$

For $\theta$ distributed according to $\mu, L(\theta)$ has a normal distribution with mean 0 and variance $a^{2} d$. Hence, using the table of standard normal distribution, we find

$$
\mu\left(|L(\theta)| \leq \frac{a}{4} \sqrt{d}\right)<\frac{1}{4}
$$

Combining this with (47), we conclude that, with $\mathbb{P}_{\mu}$-probability greater than $1 / 2$, we have simultaneously $|L(\theta)|>a \sqrt{d} / 4$ and $|\widehat{T}|<a \sqrt{d} / 8$. Hence,

$$
\sup _{\theta \in \Theta_{d}} \mathbf{E}_{\theta, 1}\left[(\widehat{T}-L(\theta))^{2}\right] \geq \mathbb{E}_{\mu}\left[(\widehat{T}-L(\theta))^{2}\right] \geq \frac{1}{128} a^{2} d
$$

where $\mathbb{E}_{\mu}$ denotes the expectation with respect to $\mathbb{P}_{\mu}$. The result now follows by letting $a$ tend to infinity.

\section{REFERENCES}

CAI, T. T. and Low, M. G. (2004). Minimax estimation of linear functionals over nonconvex parameter spaces. Ann. Statist. 32 552-576. MR2060169

CAI, T. T. and Low, M. G. (2005). On adaptive estimation of linear functionals. Ann. Statist. 33 2311-2343. MR2211088 
Collier, O., Comminges, L. and Tsybakov, A. B. (2017). Minimax estimation of linear and quadratic functionals on sparsity classes. Ann. Statist. 45 923-958. MR3662444

Golubev, G. K. (2004). The method of risk envelopes in the estimation of linear functionals. Problemy Peredachi Informatsii 40 58-72. MR2099020

GolubEV, Y. and LEVIT, B. (2004). An oracle approach to adaptive estimation of linear functionals in a Gaussian model. Math. Methods Statist. 13 392-408. MR2126747

IBRAgimov, I. A. and KHAS'MINSKII, R. Z. (1984). Nonparametric estimation of the value of a linear functional in Gaussian white noise. Theory Probab. Appl. 29 19-32. MR0739497

Laurent, B., Ludeña, C. and Prieur, C. (2008). Adaptive estimation of linear functionals by model selection. Electron. J. Stat. 2 993-1020. MR2448602

Ledoux, M. and TAlagrand, M. (1991). Probability in Banach Spaces: Isoperimetry and Processes. Ergebnisse der Mathematik und Ihrer Grenzgebiete (3) [Results in Mathematics and Related Areas (3)] 23. Springer, Berlin. MR1102015

Petrov, V. V. (1995). Limit Theorems of Probability Theory: Sequences of Independent Random Variables. Oxford Studies in Probability 4. Oxford Univ. Press, New York. MR1353441

TsYBAKOv, A. B. (1998). Pointwise and sup-norm sharp adaptive estimation of functions on the Sobolev classes. Ann. Statist. 26 2420-2469. MR1700239

Tsybakov, A. B. (2009). Introduction to Nonparametric Estimation. Springer, New York. MR2724359

VERZELEN, N. (2012). Minimax risks for sparse regressions: Ultra-high dimensional phenomenons. Electron. J. Stat. 6 38-90. MR2879672

O. COLLIER

MODAL'X

UNIVERSITÉ PARIS-OUEST

NANTERRE, 92000

FRANCE

A. B. TSYBAKOV
CREST, ENSAE
5, AVENUE HENRY LE CHATELIER
91220 PALAISEAU
FRANCE
E-MAIL: alexandre.tsybakov@ensae.fr
L. COMMINGES

CEREMADE

UNIVERSITÉ PARIS DAUPHINE

PARIS 75776 CEDEX 16

FRANCE

N. VERZELEN

INRA, UMR 729 MISTEA

F-34060 MONTPELLIER

FRANCE

E-MAIL: nicolas.verzelen@inra.fr 